\title{
La medición de impacto y evaluación de programas de alfabetización en información para bibliotecas escolares
}

\author{
Miguel Ángel Marzal*, Pablo Parra** , María Jesús Colmenero*
}

Resumen: Se analiza el planteamiento teórico, desarrollo metodológico y aproximación a unos primeros resultados de un proyecto de investigación aplicada, financiado por el Ministerio de Educación, para la propuesta de un modelo de indicadores competenciales de alfabetización en información en bibliotecas escolares. A partir de la consideración de la alfabetización en información como competencia para conocer y saber en red y la biblioteca escolar como Centro de Recursos para la Enseñanza y el Aprendizaje (CREA), se plantean, siguiendo propuestas ya enunciadas, como objetivos el diseño de indicadores cualitativos para medir el progreso y la calidad de programas de alfabetización en información para bibliotecas escolares. Se presenta la formulación del modelo de indicadores, categorizado en destrezas, habilidades y competencias, plasmado luego en un cuestionario-tipo según niveles educativos y sobre unos recursos digitales seleccionados, que fue pre-testado para su aplicación en Asturias, Navarra y Madrid. Se describe su aplicación en las tres comunidades, la naturaleza de las cuestiones, el procesamiento de datos y una aproximación a un primer análisis interpretativo por niveles educativos. Se aborda, finalmente, la argumentación de unas primeras conclusiones a modo de desarrollos futuros del proyecto y recomendaciones para el desarrollo de la Sociedad del conocimiento, a partir de un eficaz plan de programas de alfabetización en información desde bibliotecas escolares transformadas en CREA.

Palabras clave: alfabetización en información, biblioteca escolar, CREA, evaluación de programas de alfabetización en información.

\section{The measurement of impact and evaluation of information literacy programs in school libraries}

Abstract: This article analyzes the theoretical approach and methodological development of a project of applied research, as well as offering an approximation to the early results. The aim of the project, financed by the Ministry of Education, was to propose a model of indicators for information literacy competency for school libraries. In considering information literacy as a competency for knowing and learning via networks, and the school library as a Teaching and Learning Resource Center, the authors propose a design

* Departamento de Biblioteconomía y Documentación. Universidad Carlos III de Madrid. Correo-e: mmarzal@bib.uc3m.es; mcolmene@bib.uc3m.es.

** Biblioteca Municipal "Ricardo León” de Galapagar, Madrid. Correo-e: bibliogalapagar@gmail. com.

Recibido: 15-04-2010; 2. ${ }^{\mathrm{a}}$ versión: 16-11-2010; aceptado: 01-12-2010. 
for qualitative indicators to measure the progress and quality of information literacy programs in school libraries. The design of the model of indicators is presented, with the model categorized according to skills, abilities and competencies. These criteria were introduced into a questionnaire based on educational levels and covering selected digital resources. The applicability of this tool was tested in Asturias, Navarre and Madrid. The application of the questionnaire in these three regions of Spain, the nature of the questions, the processing of the resulting data, and an initial interpretative analysis by educational levels are described. Finally some initial conclusions are put forward for a future project, as are recommendations for developing a Knowledge Society beginning with an effective plan for information literacy programs in school libraries to be transformed into Teaching and Learning Resource Centers.

Keywords: information literacy, school libraries, learning and teaching resources centres, information literacy program's assessment.

\section{Introducción}

La inclusión de programas de alfabetización en información en los currículos académicos parece, cada vez más, un elemento muy positivo en la necesaria transformación del modelo educativo, hacia un carácter esencialmente competencial. La inclusión de estos programas, que suscita un considerable consenso estratégicamente, plantea sin embargo importantes dificultades tácticamente.

Debe definirse la inclusión de la alfabetización en información, dentro de las instituciones educativas regladas, en tanto que eje transversal de contenidos, materia transversal para competencias genéricas, asignatura para competencias específicas, instrumento auxiliar para e-learning, o cursos profesionales como «extensión escolar». Las universidades españolas están comenzando a dar una respuesta, variada, pero también comienza a hacerse un planteamiento en los centros educativos preuniversitarios, con una notable peculiaridad: la alfabetización en información, de la que han tomado perfecta y nítida conciencia en centros de primaria y secundaria, se enmarca siempre dentro de la esfera de la biblioteca escolar, como espacio y motor idóneo.

Enlazar la alfabetización en información y la biblioteca escolar parece una estrategia oportuna, si bien formula interrogantes específicos, que se unen a los apuntados más arriba: una biblioteca escolar que debe transformar sus funciones hacia un centro de recursos, para integrar adecuadamente el programa; el diseño curricular de un programa de alfabetización en información, que debe acreditar o certificar competencias en los educandos; una sólida formación de los «formadores", profesores con responsabilidades en la biblioteca escolar, que deben desarrollar con calidad un programa, muy transversal en su ejercicio, pero muy específico en sus contenidos; un modo y método, con instrumentos propios, para evaluar el programa, esto es, evaluar la consecución de competencias por el educando, pero también evaluar los beneficios del programa para la comunidad escolar por optimizar la calidad del proceso educativo. 
Este fenómeno ha animado a algunas instancias educativas pertinentes a tomar iniciativas. La dedicación de 2010 como Año Europeo de Lucha contra la Pobreza y la Exclusión Social, por la Unión Europea, con especial énfasis en actuar contra la proyección de la brecha digital en brechas educativas, junto con el sesgo del Informe PISA hacia la consideración de evaluar las competencias en información desde su edición de 2009, han impulsado decididamente las iniciativas. Más aún, la fuerte propensión en nuestra sociedad hacia la "cultura de la evaluación", como factor determinante de la calidad, ha colaborado para que los proyectos de desarrollo de programas de alfabetización en información dentro de las bibliotecas escolares tuviesen presente el objetivo de la calidad educativa por evaluación, tanto competencial para el educando, como programática para la biblioteca.

En este contexto, un grupo de profesores-investigadores ${ }^{1}$, entre los que se encuentran los autores de este trabajo propusieron el Proyecto Indicadores para Competencias en Información en Bibliotecas Escolares, aprobado y financiado por el Ministerio de Educación y Ciencia.

\section{Objeto del proyecto: lectura para alfabetización en información y bibliotecas escolares}

No cabe duda que la primera década del siglo xxi ha supuesto un período de franco progreso y expansión para una especialidad que damos en llamar, alfabetización en información. La especialidad, a partir de un importante elenco de iniciativas, buenas prácticas y cursos, tanto en bibliotecas como en distintos centros de formación, así como un riguroso proceso de edición de "normas", experimentó un interesante proceso de depuración en su noción. Luego las acciones de IFLA (con la Sección de alfabetización en información y la edición del sitio web InfolitGlobal) y UNESCO (Cumbres, Congresos, ediciones), la eclosión de publicaciones, congresos, proyectos y grupos de investigación, portales de información, planes competenciales para bibliotecas, iniciativas gubernamentales (caso del portal ALFARED, editado por el Ministerio de Cultura de España) han impulsado una definición, cada vez más precisa, de la alfabetización en información que se configura como la competencia clave para la eficacia del proceso educativo de los estudiantes de cualquier nivel y su capacidad para sobrevivir y progresar en una economía global de cambio, que cada vez más debilita la soberanía del Estado-nación (Bundy, 1998).

La situación actual de la alfabetización en información puede describirse como el área de las "Siete Esquinas" (conocido así en Estados Unidos para referirse al lugar creado por la unión de cinco caminos) repleta de señales a menudo con-

\footnotetext{
${ }^{1}$ Grupo liderado por Miguel Ángel Marzal, con colaboración de Rosa Piquín (Asturias), Ana Bernal y Francisco Soto (Navarra), Pablo Parra, María Jesús Colmenero, Laura Andreu, Aurora Cuevas, Amalio Sánchez y Juan Manuel Játiva (Madrid).
} 
fusas, visibilidad limitada y difícil acceso (Driscoll, 2010). La alfabetización en información tiene como campo propio las competencias de información, cuyas dimensiones son: competencia para la gestión de contenidos web, mediante una "lectura digital"; competencia en la edición cooperativa, para la diseminación ética y solidaria del conocimiento, a través de los objetos de aprendizaje; competencia evaluativa y autoevaluativa en discernir el progreso intelectivo en la selección y evaluación de contenidos para un análisis suficiente en la generación de conocimiento (Marzal, 2009). El concepto, así expresado, tiene un evidente sesgo hacia los entornos tecnológicos, lo que no obsta para que pueda tener una eficaz aplicación en entornos "gutenberg", sin embargo hay dos impulsos poderosos hacia el sesgo apuntado: la necesidad de una neta diferenciación con la formación de usuarios (se circunscribiría a la biblioteca, incluso digital, con sus instrumentos de gestión, en tanto la alfabetización en información se proyecta en los centros de recursos y gestión de contenidos en web); la imprescindible asociación de los instrumentos 2.0 a los centros de recursos y la necesidad de incorporar a toda la población al desarrollo de la Web Semántica. Cabe señalar, por lo demás, lo que el concepto de alfabetización en información debe a las aportaciones conceptuales de la ciberalfabetización y la alfabetización múltiple.

El propio desarrollo de la Web hubiera servido de motor para el progreso de la alfabetización en información como especialidad, ya que las experiencias en innovación por uso de TIC muestran estar aún en la fase de la sintaxis tecnológica, como uso hábil de herramientas TIC para conseguir determinados objetivos didácticos, cuando el propósito es que el usuario llegue a la fase de la semántica tecnológica, en la que la herramienta se transforme en un instrumento colaborativo de gestión de contenidos digitales, para generar conocimiento mediante la consecución de competencias en información. Sin embargo tres factores han convertido la alfabetización en información en un medio poderoso, lo que le ha permitido incorporarse a la agenda de las autoridades políticas y le ha proporcionado una proyección muy visible: la necesidad de combatir la brecha digital, fomentando la alfabetización en información como instrumento de inclusión social; la relevancia del aprendizaje permanente en el nuevo modelo socio-económico, para el que se hace necesario un e-learning eficaz, mediante el desarrollo por el educando de unas sólidas competencias en información; el diseño de un modelo educativo basado en competencias.

Los tres factores apuntados tienen como vector la necesidad de incorporar la alfabetización en información a las instituciones escolares, en todos sus niveles. El problema es que debe incorporarse a la escuela, en su sentido lato, sin tener una adscripción a una disciplina, ciencia o área de conocimiento, sin encaje, pues, en un Departamento escolar, sin un diseño curricular, que la defina como eje temático, materia transversal, asignatura o complemento de formación, lo que se complica si la consecución de sus competencias debe endosarse al educando como una certificación o una acreditación en su título o diploma. Estas circunstancias y su propia naturaleza informativa, han hecho que (junto a iniciativas de incorporación curricular transversal en algunas titulaciones) las bibliotecas edu- 
cativas (escolar y universitaria para la Educación Formal, pública para la Educación No Formal) se convirtiesen en su escenario lógico. El problema se agudiza porque en el sistema educativo la biblioteca carece de presencia en el currículo, los bibliotecarios tienen serias deficiencias en la docencia (los escolares por inexistencia, los universitarios por no ser considerados personal docente e investigador), se les pide formar en una especialidad para la que no han sido formados y en la que sólo perciben "buenas prácticas", pero sin una dimensión educativa real.

Cada tipo de biblioteca (cada biblioteca) está encarando el desafío con diferentes estrategias, la biblioteca escolar con mayores dificultades por razones evidentes. Sin embargo, este fenómeno que podía ser una nueva amenaza, puede convertirse en una formidable oportunidad, si se aprovecha para su saludable transformación en CREA (Marzal y Cuevas, 2007). El nuevo modelo educativo competencial desplaza las bibliotecas, los centros de recursos, (CRAI en universidad, CREA en centros preuniversitarios) al centro del proceso de enseñanza y aprendizaje, lo que otorga a la biblioteca escolar un protagonismo desconocido. El Manifiesto UNESCO/IFLA sobre la Biblioteca Escolar (2000), o las IFLA/UNESCO School Library Guidelines (2002), demuestran este ascenso a escala internacional, que se va replicando a escalas nacionales. En España, la Ley Orgánica de Educación 2/2006, de 3 de mayo, (LOE) es una clara evidencia, así como la Ley 10/2007, de 22 de junio, de la lectura, del libro y de las bibliotecas, que reconoce a las bibliotecas escolares la misión de la promoción y el fomento de la lectura, como también la apertura de las bibliotecas escolares a la comunidad de ciudadanos de su entorno, y su incorporación a las nuevas tecnologías.

Es en el ámbito autonómico, sin embargo, donde en España se está produciendo el mayor desarrollo normativo respecto de las bibliotecas escolares y alfabetización en información, lo que es perfectamente lógico por nuestra estructura institucional. Las 17 comunidades autónomas han ido desarrollando normas y planes de mejora de las bibliotecas escolares, en sus fondos, gestión y proyección, con indicación de su lógica "función formativa" (algunas normas citan expresamente la alfabetización en información), así como su modelo referencial de evolución a "centro de recursos". Sin embargo, la exégesis del corpus normativo sobre bibliotecas escolares demuestra la encomienda de dos misiones primordiales a esta biblioteca: el fomento de la lectura lúdica y comprensiva de ficción y no-ficción; y la disponibilidad, acceso y uso de recursos digitales, para los que se requieren sus propias habilidades. La publicación del portal Leer.es (http:// leer.es/) es la demostración fehaciente de la relevancia del binomio biblioteca escolar-lectura, pero también de la importancia y preocupación por las nuevas lecturas en nuevos entornos.

El Proyecto de Indicadores para Competencias en Información en Bibliotecas Escolares, por tanto, se planteaba, como objeto de investigación aplicada, la medición de la competencia lectora de los educandos en los nuevos entornos, mediante el diseño de unos cuestionarios, necesariamente derivados de la propuesta de un modelo de indicadores, cuyo escenario educativo fuese la biblioteca 
escolar. Los instrumentos de medición, naturalmente, debían tener un sesgo cualitativo por la naturaleza competencial del campo evaluado. La lectura digital y multifuncional en web, como elemento básico de una nueva alfabetización se convertía en el fundamento del proyecto, por cuanto reclama modos de medición de progreso competencial, determinantes del desarrollo de programas de alfabetización en información y la transformación de la biblioteca escolar en CREA.

\section{Objetivo: la evaluación lectora para programas de alfabetización en información en bibliotecas escolares}

La lectura, desde luego también en la óptica de la alfabetización en información, es producto de una destreza y una habilidad, que integran en el educando un compendio de aptitudes, actitudes y capacidades, para derivar en una competencia, que faculta al educando para generar aptitudes y capacidades de conocimiento, que se ponen en acción para la resolución de un problema concreto en un contexto concreto, dando origen a un saber que se orienta hacia la decisión y acción más eficaces.

La alfabetización en información tiene una naturaleza competencial para el conocimiento y saber, que precisa, en cambio y por sus propiedades, un diseño evaluativo propio, que contemple el proceso evaluativo desde dos dimensiones: la evaluación, modo de medir la eficacia de un programa de alfabetización en información en generar conocimientos y competencias en los educandos, conforme a sus objetivos; la valoración, para la medición de actitudes, valores y habilidades adquiridas en el programa. Los programas de alfabetización en información deben integrar ambas dimensiones mediante una "evaluación programática" para la biblioteca escolar ("evaluación") a través de indicadores y una "evaluación formativa" para los educandos ("valoración"). Así se consideró en el proyecto.

La alfabetización en información ya cuenta con importantes antecedentes en evaluación, como la tipificación de la IFLA (evaluación diagnóstica, formativa y sumativa), los aspectos evaluables de la ACRL (evaluación de programas y profesorado, evaluación del aprendizaje, la transferibilidad de buenas prácticas), o los criterios evaluativos de las mejores prácticas por el Information Literacy Institute (programas, logros, programas-logros). Lindauer, por su parte aportó los tres ámbitos propios de la evaluación de la alfabetización en información, esto es, el entorno de aprendizaje (planes de estudios), los componentes del progra$m a$, referido a la existencia de las oportunidades, y su alcance y los resultados de aprendizaje, sobre el rendimiento de los educandos (Lindauer, 2006).

Un sistema de medición de impacto de un fenómeno y su evaluación precisa de la definición de unas categorías, que actúan como el contexto de interpretación de los datos proporcionados por los futuros indicadores, junto con unos procedimientos, consistentes en los métodos de aplicación de las categorías y sus indicadores. La naturaleza de las competencias en información, siguiendo la concepción arriba anotada, aconsejaba que las categorías se estructurasen en una 
escala progresiva de destrezas, habilidades y competencias, cada una de ellas con sus propios indicadores, para una adecuada medición del impacto competencial. Esta estructuración escalable en la definición de un modelo de medición de impacto de alfabetización en información en bibliotecas escolares conduce al empleo de "métodos cualitativos" por su mayor eficacia para evaluar actitudes, valoraciones y motivaciones (Viñas, 2004).

El campo propio de la alfabetización en información, pero también los caracteres de la competencia lectora en los nuevos entornos tecnológicos dirigen el espacio de acción educativa hacia un estilo de e-learning, por lo que son útiles sus enfoques evaluativos del e-learning, respecto a los beneficios sobre la comunidad escolar y la eficacia en la construcción del aprendizaje por el educando por la interacción con unos contenidos educativos digitales, pero sobre todo sus principios evaluativos, susceptibles de modelar indicadores (Colás et al., 2005), especialmente la interiorización (apropiación de las tecnologías), la privilegiación (capacidad de seleccionar la TIC idónea para conocer) y reintegración (capacidad de dominar el "lenguaje» de la TIC para su óptima aplicación).

La evaluación se proyecta en unos instrumentos de medición de impacto. Sin duda, en el entorno de las bibliotecas escolares, un programa competencial en información reclama dos tipos como idóneos: los cuestionarios para la valoración, por procesar eficazmente las percepciones; los indicadores para procesar eficazmente factores estadísticos, entendidos como estimadores para la medición de variables o condiciones determinadas y así analizar un fenómeno y su evolución. La gran variedad de fenómenos, objetivos, propósitos, finalidades evaluables, explica que los indicadores no son una realidad monolítica, sino que tienen su tipificación, según la naturaleza de la evaluación. Sin duda para programas competenciales en información son convenientes indicadores de diagnóstico, para evaluar deficiencias y disfunciones, indicadores de seguimiento, para evaluar y mejorar el proceso, indicadores de resultados, para verificar el cumplimiento de los objetivos y evaluar sus beneficios.

Así pues, la alfabetización en información cuenta con herramientas evaluativas específicas, con su propia metodología (Emmet y Ende, 2007), así como programas, entre ellos, SAILS (Standardized Assessment of Information Literacy Skills), basado en las normas de la ACRL y la AASL, la prueba iSkills de la empresa Educational Testing Services, por su parte, desarrolló la encuesta Information Skills Survey de CAUL (basado en las normas CAUL/ANZIIL), con el objetivo de detectar el nivel competencial de los educandos en áreas académicas específicas y se destinaba para la toma de decisiones. En España se apunta el proyecto ALFIN-HUMA, dirigido por M. Pinto, con una clara proyección en el ámbito académico universitario.

Como una respuesta global a estas iniciativas debe considerarse la publicación de R. Catts y J. Lau, Towards Information Literacy Indicators, por la UNESCO en París, 2008. El proyecto se plantea como la propuesta de un conjunto de indicadores para medir las habilidades en información, a partir de indicadores ya diseñados y que habían demostrado una cierta validez evaluativa, como los pro- 
gramas LAMP, PISA y cuestionarios de Instituto de Estadísticas de UNESCO, OCDE, DHS o la OIT.

\section{Justificación}

A la hora de evaluar la aplicación de programa de alfabetización en información en unidades de información, existe una tendencia de emplear indicadores cuantitativos. Por la propia naturaleza de estos indicadores, se observa que en la mayoría de las experiencias de aplicación de este tipo de herramientas a programas de alfabetización en información se dirigen a la medición y a la evaluación de acciones en función del uso, pero por lo general no se trata propiamente de indicadores competenciales. Lo mismo sucede con los indicadores aplicados al uso tecnológico en el ámbito educativo que suelen medir parámetros como el porcentaje de personas que usan el ordenador para el aprendizaje, número de equipos informáticos destinados a profesores y alumnos, con conexión a Internet, frecuencia de uso, etc. El último ejemplo es el informe Indicadores y datos de las Tecnologías de la Información y Comunicación en la Educación en Europa y España presentado por el Instituto de Tecnologías Educativas (ITE), unidad del Ministerio de Educación responsable de la integración de las TIC en las etapas educativas no universitarias. En él se muestran brevemente algunos indicadores de las TIC en los 27 países de la Unión Europea y en el sistema educativo español y se analiza la situación de los centros públicos y privados españoles no universitarios respecto a las TIC a partir de dos fuentes: los resultados de un estudio realizado por la Fundación Telefónica y la Estadística de la Sociedad de la información y la comunicación en los centros educativos 2007-2008 del Instituto Nacional de Estadística (INE).

La propensión hacia "indicadores cualitativos" conecta con el impulso que la Unión Europea está otorgando a la medición e impacto de las TIC, a partir de los planes eEurope 2002 y 2005, desde una perspectiva no sólo, ni prioritariamente, infraestructural. Demostración palpable se obtiene del análisis de la tabla de indicadores de eEurope 2010, en la que se reconocen como categorías la alfabetización digital, aprendizaje y formación, brechas digitales. La constitución de la Asociación para la medición de las TIC para el desarrollo (Partnership on Measuring ICT for Development), con el objetivo de crear una plataforma de convergencia de los distintos modelos de indicadores para hacerlos compatibles en un análisis interpretativo comparado útil, no hace sino confirmar esta propensión, de la que se hizo eco el proyecto. El refrendo a esta necesaria evolución procede, por fin, de los indicadores propuestos para i2011-2015, recogidos por el i2010 High Level Group, a finales de 2009.

De otro lado, la propia concepción de las competencias requieren instrumentos válidos para evaluar, especialmente porque el Informe PISA, así como las pruebas competenciales que, en el caso de España están desarrollando algunas autonomías, pretenden contar con datos fehacientes sobre la calidad y progreso 
del modelo educativo. En la investigación a la que se refiere el presente proyecto, pues, la evaluación tiene dos vertientes necesarias de aplicación: la alfabetización en información y la importancia concedida a las bibliotecas escolares.

En el primer caso, la acción educativa se concreta en el desarrollo competencial atendiendo a tres dimensiones para una eficaz captación intelectiva de contenidos digitales: la captación selectiva, distribuida y sostenida en la usabilidad de los recursos electrónicos; la fidelización del recurso para la asimilación eficaz de su contenido; y la capacidad alfabetizadora del recurso. A partir de la consideración de esta triple dimensión educativa, el diseño evaluativo para la medición de progreso a través de la alfabetización en información en el entorno escolar debe reconocer distintos niveles en su programación:

- Medición de impacto en el consumo de información educativa, como diagnóstico de deficiencias competenciales.

- Medición del nivel de adquisición de destrezas en un uso eficaz de las herramientas de lectura y escritura digitales.

- Medición del nivel de adquisición de habilidades en el uso eficaz de instrumentos de gestión de contenidos para una lectura y escritura digitales comprensivas.

- Medición del nivel de adquisición de competencias para la organización y representación de conocimientos en web, mediante edición de conocimientos, acreditación de un saber competencial adquirido.

- Evaluación continua y autónoma del programa, por parte del mismo educando mediante ejercicios de autoevaluación, así como un modelo de evaluación práctica para los docentes sobre las competencias adquiridas.

- Procesamiento de los indicadores dentro de un modelo de evaluación, capaz de generar informes evaluativos sobre la calidad y excelencia del programa para los educandos y para la institución que los gestiona.

El proyecto, pues, se diseñó para la obtención de un cuestionario inicial de diagnóstico de deficiencias competenciales, y un modelo de indicadores para su procesamiento analítico. El modelo, necesariamente, debía ser escalable, por la propia dinámica de consecución de competencias y por el propio espectro de los educandos, sometidos a profundos cambios de madurez intelectiva durante todo su proceso escolar.

La segunda vertiente de aplicación del proyecto se refiere a la importancia concedida a las bibliotecas escolares en las competencias lecto-escritoras. Es evidente que a las bibliotecas escolares se les concede tanta importancia ideal y teórica en el contexto educativo, como se la ningunea en la realidad y práctica cotidianas. Sin embargo, no es menos cierto que distintos factores están contribuyendo decisivamente en la emergencia de la biblioteca escolar como factor educativo: los conocimientos competenciales transversales que tienen como espacio idóneo la biblioteca escolar, los planes de fomento a la lectura (nacional y autonómicos), que dirigen sus disposiciones hacia la biblioteca escolar como 
vehículo de estímulo y ejercicio, la imprescindible transformación de la biblioteca escolar en centro de recursos para el aprendizaje y la enseñanza, cuyos elementos definitorios son el desarrollo de una colección digital educativa, programas de alfabetización en información, la incorporación de la web 2.0 y redes sociales. El proyecto se debía dirigir, pues, a la generación de instrumentos que midiesen el progreso en esta necesaria y saludable transformación.

\section{Metodología y recursos empleados}

\subsection{Formulación del modelo aplicativo}

El punto de partida del proyecto de investigación estribó en una labor de reconocimiento y compilación de fuentes de información relevantes y suficientes para determinar el "estado del arte" sobre modelos de evaluación de competencias informativas para la lectura, especialmente en el ámbito digital, espacio propio de la alfabetización en información. La recopilación de fuentes supuso un posterior análisis de objetivos y efectividades aplicativas, así como análisis comparativo de las estrategias, tutoriales y productos evaluativos resultantes. El proceso derivó en el establecimiento de un conjunto de requisitos competenciales que consecuentemente determinaron un Modelo de Indicadores de Competencia Informativa para evaluar la eficacia del aprendizaje mediante una lectura para la inclusión educativa.

El modelo resultante es escalable por cuanto se adapta al nivel cognitivo de cada educando y se aplica en su totalidad o en las partes que se estimen oportunas, estructurándose en tres categorías conforme a un esquema de capacidades:

- Categoría de Indicadores de Destrezas, referidos fundamentalmente a las capacidades del lector respecto a la accesibilidad y usabilidad tecnológicas de los recursos que son leídos. El protagonismo radica en la interacción del lector con el recurso.

- Categoría de Indicadores de Habilidades, referidas a las capacidades del lector en la obtención de un producto cognitivo, un conocimiento y un saber, por dominio de una gramática del discurso. El protagonismo está en el dominio por el lector del proceso inferencial de la lectura para generar conocimiento.

- Categoría de Indicadores de Competencias, referidas a las capacidades del lector resultantes de un dominio de las normas de alfabetización en información. El protagonismo corresponde completamente al usuario-educando, por lo que se evalúan sus competencias en su autonomía de aprendizaje permanente, con mecanismos, valores y deontología propios.

A continuación, se generó una plantilla definida en 28 indicadores, a partir de los cuales se creó una herramienta de evaluación de las destrezas, habilidades 
y competencias informativas de los alumnos con respecto al uso de los recursos de información educativa. La herramienta, materializada en forma de cuestionario, se confeccionó atendiendo a criterios de evaluación descriptiva y predictiva e incluía evaluación basada en conducta, evaluación basada en la opinión y evaluación de usabilidad. El cuestionario estaba formado por preguntas cerradas y abiertas, con un carácter marcadamente cualitativo, imprescindible en una evaluación de competencias.

\subsection{Recolección de datos y muestreo}

El propósito principal en esta etapa se centró en la determinación de una metodología propia que permitiera evaluar la eficacia y validez de los indicadores desarrollados, a fin de poder elaborar unas recomendaciones que orienten futuras directrices de actuación enfocadas a la consolidación del proceso de transformación de las bibliotecas escolares en CREA. Para llevar a cabo su ejecución se aplicaron los indicadores diseñados en un entorno real (una muestra de alumnos de tres comunidades autónomas: Asturias, Madrid y Navarra) con el fin de validarlos, por un lado, y detectar, diagnosticar y sugerir acciones para la competencia lectora, por otro. El hecho de llevarse a cabo en varios espacios geopolíticos distintos de nuestro país permitía además establecer un método comparativo complementario para observar si existen o no diferencias significativas entre sus contextos educativos. La elección de las Comunidades Autónomas se justifica en sus marcos normativos y en la visibilidad en torno a su acción educativa y al movimiento de sus bibliotecas escolares.

Desde el inicio del proyecto se planteó que el universo estadístico objeto del estudio debía estar formado por escolares que cursaran en España estudios de formación no universitaria, tanto obligatorios (ciclos formativos de Primaria y Secundaria) como no obligatorios (Bachillerato y Formación Profesional de Grado Medio y de Grado Superior) separados por grupos de edad, estableciéndose como muestra más adecuada los alumnos de centros públicos y concertados pertenecientes al curso final de cada etapa formativa:

- Primaria: para la evaluación de los alumnos en el último curso, con 12 años, correspondiendo a un desarrollo de competencias incipiente.

- ESO y Ciclo Formativo de Grado Medio: para la evaluación de los alumnos en el último curso, con 16 años, desarrollo avanzado.

- Bachiller y Ciclo formativo de Grado Superior: para la evaluación de los alumnos en el último curso, con 18 años, desarrollo experto.

De acuerdo a la escalabilidad del modelo, se consideró que debían diseñarse tres cuestionarios diferentes, uno por cada etapa formativa, con un número de entre 40 y 50 preguntas agrupadas en dos grandes bloques. El primero estaba compuesto por una batería de preguntas directas sobre el conocimiento previo 
del alumno de las herramientas TIC y su relación con el uso educativo. El segundo bloque incluía preguntas directamente relacionadas con el contenido de los recursos educativos propuestos en cada caso y elegidos por los diez miembros del grupo de profesores-investigadores en su condición de expertos sobre la materia. Los recursos seleccionados para cada nivel fueron los siguientes:

- Primaria: Educalia Infantil y Primaria ${ }^{2}$. Es un programa educativo que la Fundación Telefónica desarrolló en Internet para la comunidad que forman los niños y jóvenes de 3 a 12 años, sus familias y sus profesores. Se basa en una concepción amplia de la educación y se caracteriza por ser una web abierta, interactiva y dinámica ya que sus contenidos aumentan, progresan y mejoran con la colaboración de todos.

- ESO y Ciclo Formativo de Grado Medio: En un lugar de la red re $^{3}$ Se trata de una aplicación interactiva multimedia creada por Lluís Rius Oliva para dar a conocer y estimular la lectura del Quijote. Esta guía de lectura interactiva aspira a ser un pretexto ideal para promover el interés del alumnado hacia esta obra clásica y contribuir a romper la tradicional animadversión de los adolescentes hacia los textos escritos.

- Bachiller y Ciclo formativo de Grado Superior. Historia de la filosofía ${ }^{4}$ Sitio web creado en octubre de 2001 por José María Fouce, profesor de Filosofía en la actualidad del I.E.S. Salvador Dalí de Leganés (Madrid). Su principal objetivo es proporcionar a los alumnos un conjunto de elementos complementarios de los que reciben en clase, así como ofrecer un resumen con los contenidos más significativos del pensamiento de los filósofos.

Los cuestionarios fueron sometidos en los meses de febrero y marzo de 2009 a un total de 839 alumnos de veinte centros de enseñanza ${ }^{5}$ de Asturias, Madrid y Navarra a lo largo de dos sesiones. En la primera los estudiantes consultaron por su cuenta el recurso, mientras que en la segunda cumplimentaron un cuestionario a través de la plataforma telemática Moodle.

${ }^{2}$ Disponible en: http://www.educared.org/global/educalia-y-comunidad-virtual/ [consulta: 15-04-2011].

3 Disponible en: http://concurso.cnice.mec.es/cnice2005/54_en_un_lugar_de_la_red/en\%20un\% 20lugar\%20de\%20la\%20red/index.html [consulta: 13-02-2011].

${ }^{4}$ Disponible en: http://www.webdianoia.com [consulta: 13-02-2011].

${ }^{5}$ Los centros educativos que colaboraron en el proyecto y que formaron la muestra del estudio fueron los siguientes. En Asturias: Colegio Público "Baudilio Arce" (Oviedo), Colegio Público "Guillén Lafuerza" (Oviedo), Colegio Público "Elena Sánchez Tamargo" (Pola de Laviana), Colegio Público "Pablo Iglesias" (Soto de Ribera), IES "N 5" (Avilés). En Madrid: Colegio Público "Claudio Vázquez" (Morata de Tajuña), IES "Beatriz Galindo" (Madrid), IES "Complutense" (Alcalá de Henares) e IES "Alonso de Avellaneda" (Alcalá de Henares). Y en Navarra: Colegio Público "D. a Álvara Álvarez" (Falces), Colegio Público Ángel Martínez Baigorri (Lodosa), Colegio Público "San Francisco Javier" (Mendavia), IES "Marqués de Villena" (Marcilla), IES "Navarro Villoslada" (Pamplona), IES "Sierra de Leyre" (Sangüesa) e IES "Zizur" (Zizur Mayor). 


\section{Resultados y discusión}

Finalmente, se analizaron los datos obtenidos a fin de obtener conclusiones que nos permitieran evaluar el proceso y las actuaciones a adoptar. Por lo general, se observa que los resultados en general son coherentes con lo que se aprecia en las aulas. Las tecnologías se utilizan como medio lúdico y no pedagógico, manejando los alumnos el medio pero no su contenido.

Las preguntas seleccionadas para constituir el cuestionario que finalmente se sometió a los alumnos de las tres comunidades autónomas, por niveles educativos, estuvieron conscientemente sesgadas en dos planos de análisis, tal y como se apuntó anteriormente al detallar la metodología seguida:

- Preguntas para detectar la percepción que el alumno tiene sobre su propia habilidad y conocimiento en entornos tecnológicos, como también preguntas que permitiesen, en el análisis posterior, comprobar que la propia percepción se contrasta con una realidad.

- Preguntas en las que el alumno es analizado no tanto como usuario hábil en TIC, sino como "educando", esto es, que permitiesen constatar el grado de aprendizaje eficaz sobre un recurso Web educativo, a partir de escritura y lectura digital.

Teniendo en cuenta estos considerandos de análisis, se procedió a realizar una valoración para la indicación de posteriores recomendaciones, agrupadas según los tres niveles educativos elegidos.

\subsection{Primaria}

\section{Preguntas sobre uso y actitud frente a instrumentos colaborativos}

De las respuestas se deduce que los alumnos emplean las herramientas de la web 2.0, pero que no lo hacen, de forma colaborativa, en el proceso de aprendizaje en la misma medida en la que parecen que las utilizan fuera del ámbito escolar (figura 1).

\section{Preguntas sobre eficacia en la «lectura digital» (recuperación y organización de la información digital)}

Más de dos tercios de los alumnos dicen, genéricamente, que les gusta leer pero no discriminan el tipo de lectura, evidenciando que la lectura se asocia a la "escuela" y es un acto dirigido.

\section{Preguntas sobre eficacia en "escritura digital» (reconocimiento de babilidad en procesar, reproducir, difundir, editar información digital)}

Los alumnos se sienten capacitados para difundir información en red, y aplicar el contenido del recurso web a otras asignaturas y hacer un esquema de la información contenida en él, pero apenas la mitad fue capaz de hacer un resumen del contenido (figura 2). 
FIGURA 1

¿Te parecen adecuados o útiles los foros para mejorar el acceso a los contenidos?

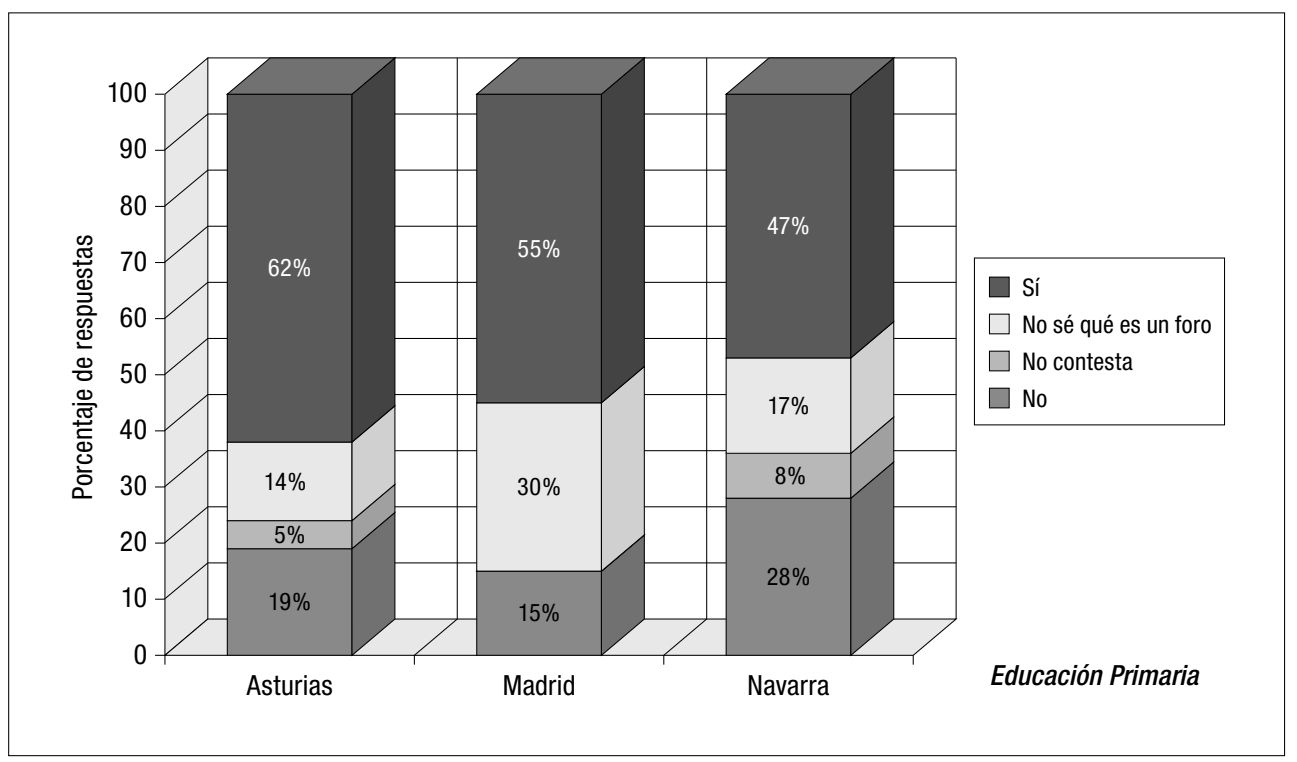

FIGURA 2

¿Sabes modificar páginas web o has becho alguna?

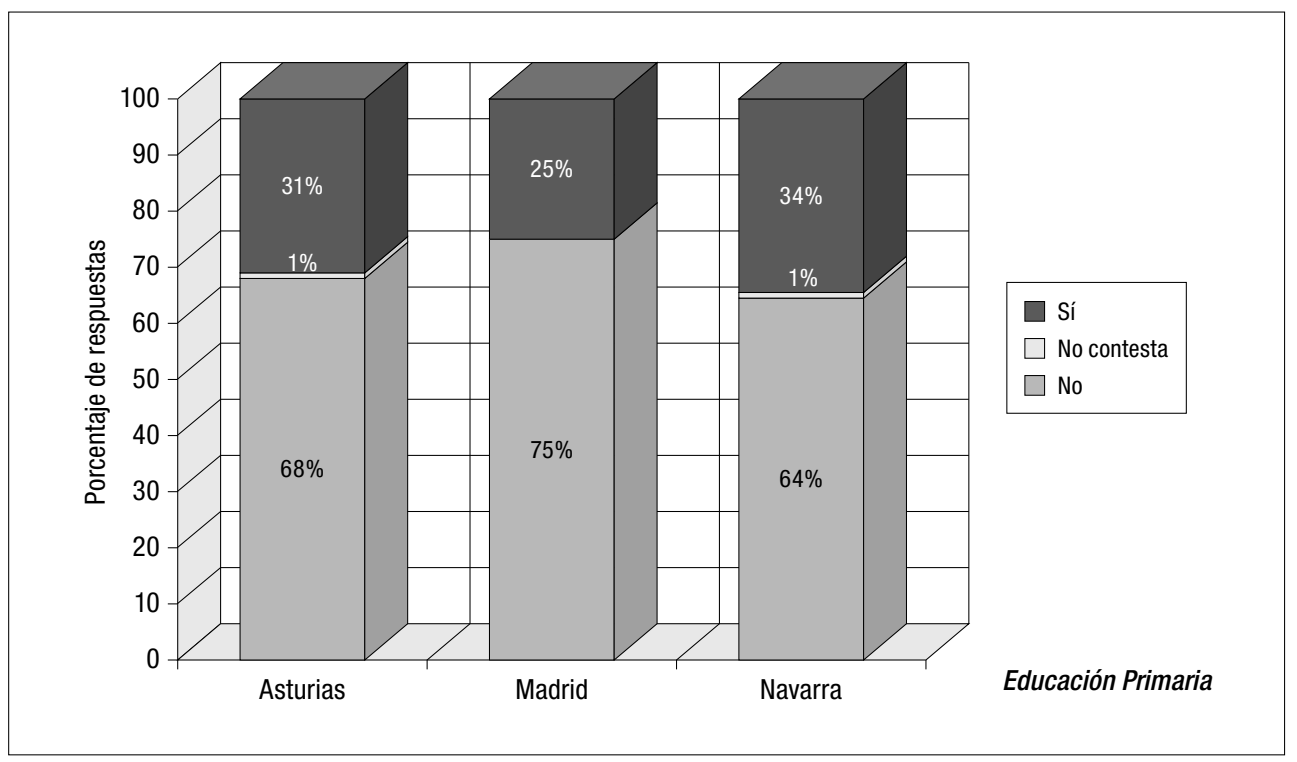


Preguntas sobre ética y deontología en red (uso ético de la información)

Los alumnos mayoritariamente afirman ser sinceros en un entorno tecnológico y son conscientes de las prohibiciones sobre copias ilegales, por lo que tienden a no copiar y pegar.

\section{Preguntas sobre uso eficaz de un recurso educativo web}

La motivación de los alumnos hacia este tipo de recursos es muy elevada, sin embargo, se comprueba que el aprovechamiento, uso y disfrute intelectual del recurso es muy superficial.

\subsection{Secundaria}

Preguntas sobre uso y actitud frente a instrumentos colaborativos

Los alumnos manifiestan un conocimiento alto y cierto sobre "redes sociales", si bien netamente ajenas al mundo educativo. También muestran el mismo sesgo pasivo en el uso de la web 2.0, así como una clara renuencia a compartir conocimiento.

\section{FIGURA 3}

¿Qué redes sociales empleas?

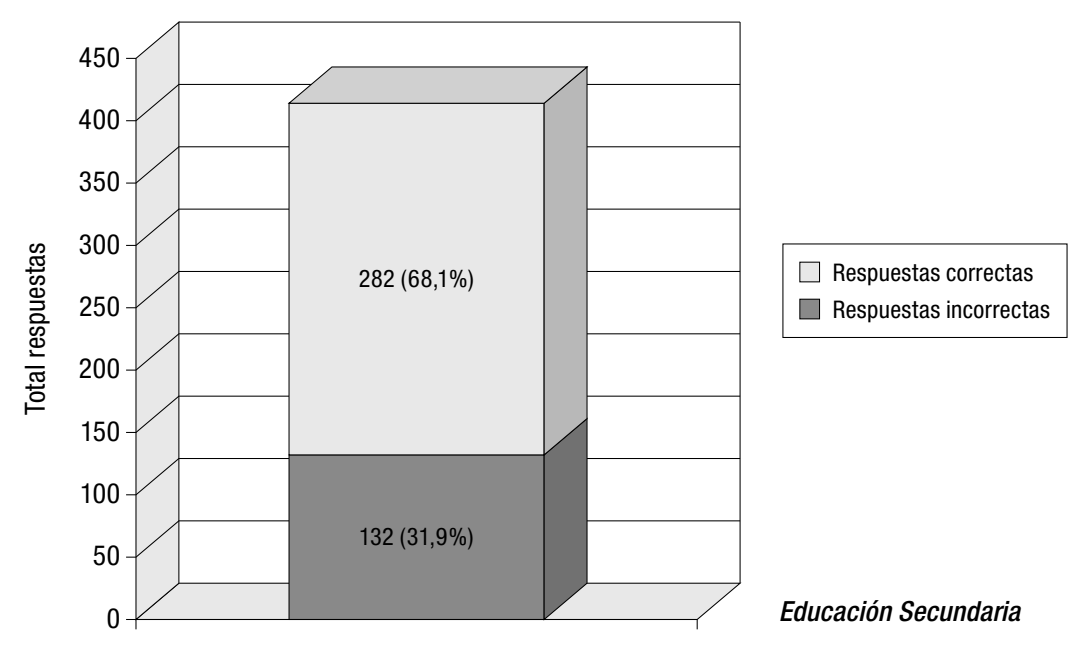




\section{Preguntas sobre eficacia en la «lectura digital»}

En este nivel educativo se hace patente una mayor habilidad en la lectura informativa de los recursos web. Además hay una menor propensión a imprimir la información recuperada y una cierta preferencia por las enciclopedias virtuales.

\section{Preguntas sobre eficacia en "escritura digital»}

Respecto a la edición es muy significativo que una mayoría de alumnos se manifiesten hábiles en hacer un esquema del contenido, pero menos de la mitad afirman interés en cambiar los contenidos del recurso.

\section{Preguntas sobre ética y deontología en red}

Los alumnos prestan fiabilidad a la información recuperada en buscadores, manifestando lógicamente que no son críticos con la información volcada. Éticamente no son respetuosos con la propiedad intelectual en red, pero curiosamente se reconocen sensibles a la intimidad de los otros en red.

\section{Preguntas sobre uso eficaz de un recurso educativo web}

La disociación entre la propia percepción de habilidad en red y su constatación manipulando un recurso concreto se evidencia. Los alumnos acreditan que el recurso es sencillo, pero las respuestas son poco certificadoras de la eficacia en el aprendizaje.

\section{FIGURA 4}

¿Has necesitado conocimientos previos para emplear esta web?

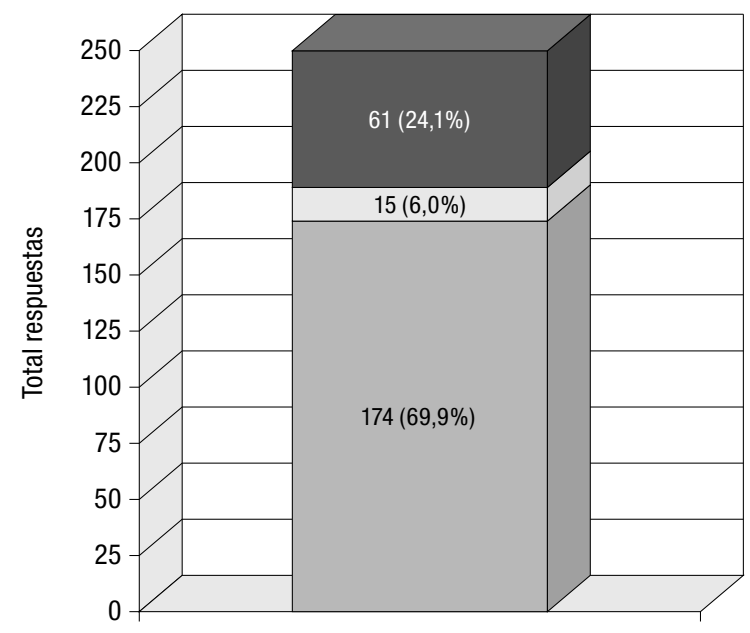

$\square$ Sí

$\square$ No contesta

$\square$ No

Educación Secundaria 


\subsection{Bachillerato-Ciclos Formativos de Grado Superior}

\section{Preguntas sobre uso y actitud frente a instrumentos colaborativos}

Hay un repunte claro en la participación en redes educativas y actividades grupales en red, lo que ratifica esta propensión extracurricular a un modelo educativo compartido.

\section{FIGURA 5}

¿Has organizado alguna vez actividades grupales en red?

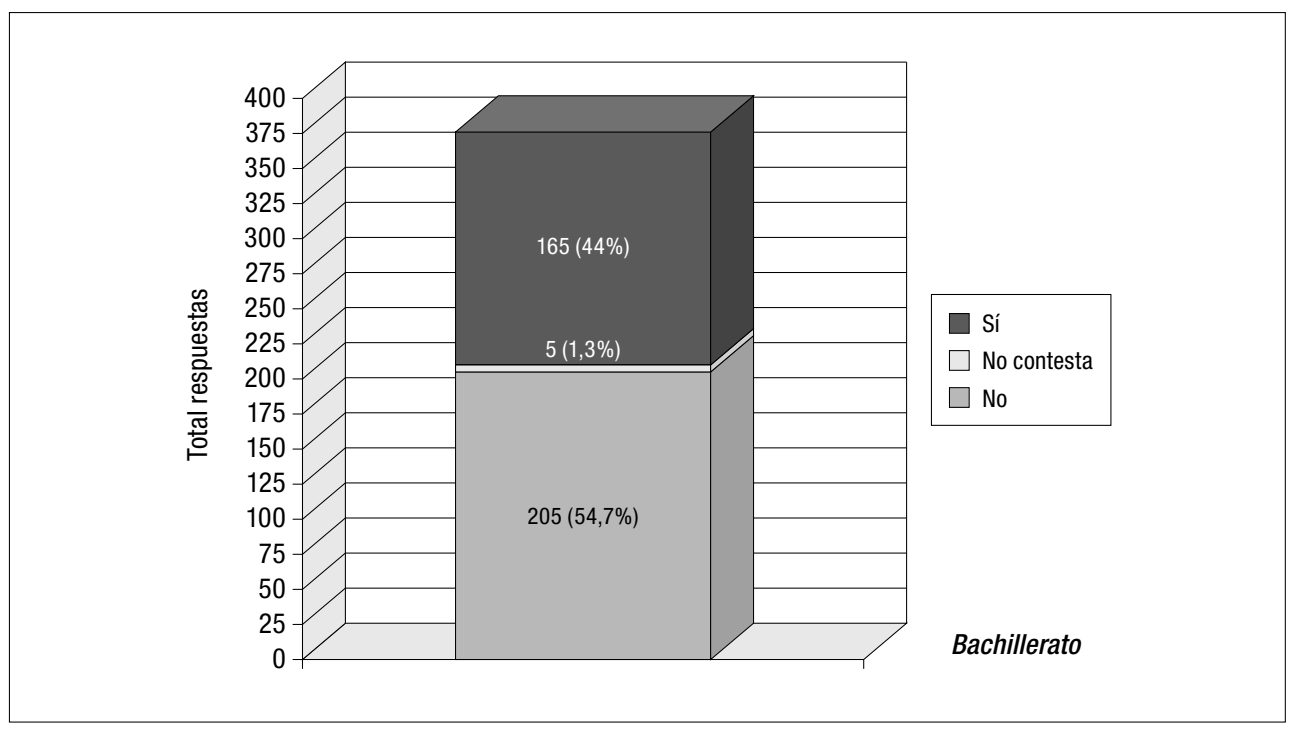

Preguntas sobre eficacia en la «lectura digital»

El alumno se percibe capaz de seleccionar los contenidos de un recurso por comprender adecuadamente su estructura, de detectar palabras clave y planificar el contenido para ser apoyo en su proceso de aprendizaje. El uso efectivo del recurso propuesto, tamiza, de nuevo, tan bonancible percepción.

\section{Preguntas sobre eficacia en "escritura digital»}

Los alumnos, en proporción suficiente, se manifiestan competentes en bloqueo-desbloqueo de contenidos en recursos web, edición de un sitio web, actuar frente a ventanas publicitarias emergentes, pero la proporción desciende ante el reconocimiento de capacidad en crear un mapa conceptual y elaborar un resumen del contenido. 


\section{Preguntas sobre ética y deontología en red}

Aun cuando son escrupulosos en el respeto a la intimidad en red y en respetar las normas de las comunidades virtuales a las que acceden, los estudiantes se muestran poco proclives al respeto de la propiedad intelectual en la web.

\section{FIGURA 6}

¿Sabes el peligro en que incurres cuando realizas copias ilegales? ¿Haces caso de los anuncios relativos a la probibición de copiar archivos de forma ilegal?

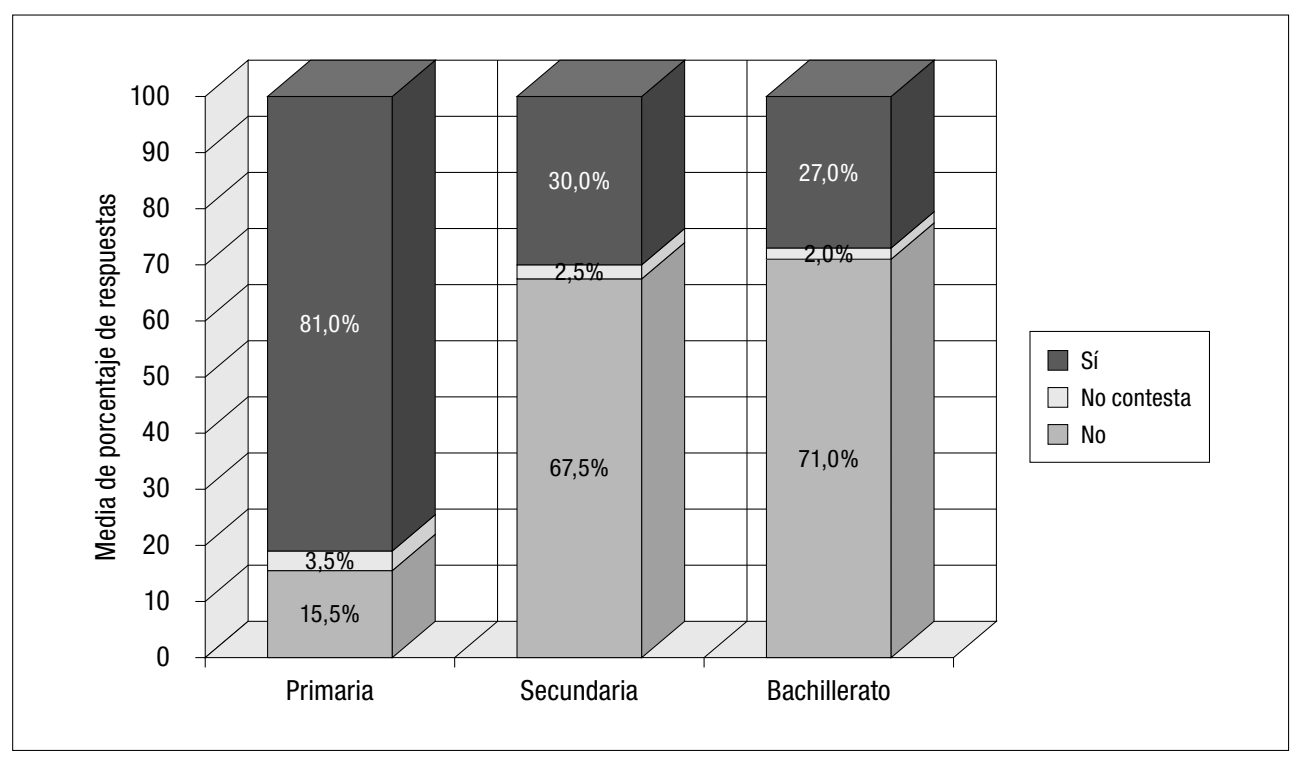

\section{Preguntas sobre uso eficaz de un recurso educativo web}

Nuevamente las preguntas certifican que el alumno percibe el recurso como fácil, interesante, intuitivo, útil, pero las respuestas certifican el conocimiento superficial de los modos de procesar el contenido digital.

\section{Conclusiones y desarrollos futuros}

A partir de las valoraciones anteriores, y de forma conjunta para todos los niveles educativos, se reflejan esquemáticamente, las siguientes recomendaciones:

\section{- Uso de instrumentos web 2.0}

Es recomendable iniciar la incorporación de este método de aprendizaje en las aulas cuando se utilicen las TIC como medio didáctico. Existen programas muy 
atractivos y los resultados de las TIC para un aprendizaje eficaz en la Web serían evidentes. Hay, por tanto, un absoluto desconocimiento del aprendizaje colaborativo, como muy probablemente la ausencia de su inoculación como método didáctico. Una comprensión real del aprendizaje colaborativo en entornos 2.0 permitiría el desarrollo educativo coherente de las aulas extendidas, esto es, la incorporación de la web como entorno educativo y la conexión de la "escuela" al ciberespacio, otorgando una dimensión de conocimiento que ahora carece la información digital, en la que tantos esfuerzos y recursos se orientan, sin embargo.

\section{- Lectura digital}

Se recomienda ejercitar la lectura de narraciones, cuentos, ejercicios, en el entorno tecnológico, para aprovechar la inclinación del alumno, que "percibe" la "facilidad" del recurso (otra cosa será la realidad), lo que es una seria "motivación", pero con técnicas propias: no se pueden utilizar una dinamización lectura igual en entorno tecnológico que en gutenberg, tal como se recomienda respecto a las imágenes, insistiendo en técnicas de "lectura icónica". La lectura digital implica no sólo una competencia en la comprensión lectora, sino en el reconocimiento de elementos "alfabetizadores" que son propios de la red, que tiene su propia semiótica y lenguaje de signos. Una correcta comprensión de éstos, capacita en una nueva alfabetización.

\section{- Escritura digital}

Se recomienda introducir metódicamente en las aulas los instrumentos y técnicas existentes y en formidable desarrollo, de instrumentos para representar y editar información, organizada para su asimilación eficaz en conocimiento. Se recomienda ejercitar en una selección crítica de los contenidos en red, esto es, un sistema serio y coherente de "evaluación" de contenidos por el educando. Se hacen imprescindibles la edición de indicadores propios y sustantivos de la evaluación de contenidos en red, que son los que deberán generar el propio vocabulario en red.

\section{- Uso y difusión ética de información}

Se recomienda incorporar técnicas de ejercicio que garanticen y optimicen el proceso de inferencias cognitivas desde la información digital a conocimiento, así como ejercitar en técnicas para búsqueda y edición de información, conforme a criterios normalizados (técnicas documentalistas), conforme a patrones deontológicos de uso de información y edición y difusión éticas de la misma. Se hace patente, en todo caso, una visible despreocupación en los usos éticos de la información y el respeto por la propiedad intelectual: son aspectos que un sistema educativo, no puede permitirse en modo alguno, en la formación de sus ciudadanos. Se hace urgente e imprescindible un aprendizaje en estos valores. 


\section{- Uso eficaz de contenidos educativos digitales}

Se recomienda avanzar en una línea que introduzca los recursos web educativos como material didáctico de primera magnitud, generar colecciones digitales constituidas por estos recursos (con sus propios modos de organización en biblioteca digital educativa), avanzar hacia la elaboración de objetos de aprendizaje, incorporar programas sólidos de alfabetización en información para un uso verdaderamente eficaz y eficiente de estos recursos en un modelo educativo competencial. Es sumamente importante que el profesorado (y las autoridades académicas) interioricen que estos recursos son insoslayables, que deben ocupar una posición de primer rango en la formación del profesorado, en los planes de lectura y en la biblioteca escolar, centro de aprendizaje con recursos más que centro de recursos para el aprendizaje.

Del proyecto Indicadores para Competencias en Información en Bibliotecas Escolares se deriva que la información obtenida a partir de los cuestionarios trabajados y los indicadores que se apuntan, certifica que se hace necesario, como línea de acción para una política de información en Educación:

a) La transformación de la Biblioteca Escolar en CREA, como instrumento insoslayable donde ejercitar la lectura y escrituras digitales, desarrollar el espacio 2.0 mediante una biblioteca 2.0, inculcar el uso ético de la información y generar, así como tratar y organizar la información digital educativa y los objetos de aprendizaje. Conviene tener muy presente que la biblioteca no es la que transforma a los usuarios, los usuarios son los que transforman la biblioteca si se le da un uso correcto. Cuanto más impersonal es el tono que empleamos para tratar sobre la función de la biblioteca, menos implicado se siente el personal.

b) La incorporación de programas formativos en alfabetización en información desde el CREA en la escuela, un programa para el que pueden contemplarse distintas modalidades, como una materia más impartida en el CREA, unos contenidos transversales incorporados en las materias como unidad didáctica, o un módulo formativo a partir de técnicas de e-learning. La gran riqueza de nuestras bibliotecas escolares (en toda España) es precisamente intentar que en ellas se desarrolle, desde otra práctica educativa que tenga en cuenta los aspectos documentales, el currículo de todas las áreas a través de metodologías activas. No se trata de un parche o una unidad didáctica, sino de entender que los aspectos documentales son necesarios en la Sociedad de la información y han de ser desarrollados desde la biblioteca escolar.

c) Definición de las competencias digitales necesarias en Educación preuniversitaria, su contemplación o auxilio a las competencias contempladas ya en el Informe PISA y reconocimiento de Indicadores que debe gestionar el Observatorio de la Lectura y Libro Infantil y Juvenil. Precisamente uno de los valores más indudables, evidentes y enriquecedores de este 
Proyecto, es que de él puede hacerse, con extrema facilidad y coherencia, una definición de competencias y una aplicación de los Indicadores que propone. De modo que se ha de señalar como una necesidad imperiosa la formación del profesorado en todos los aspectos documentales complementarios con los aspectos didácticos de las distintas áreas.

\section{Agradecimientos}

Este proyecto ha sido financiado por el Ministerio de Educación y Ciencia a través de la ORDEN ECI/754/2008, de 10 de marzo, por la que se concedían ayudas para la elaboración de materiales para facilitar la lectura en las diferentes áreas y materias del currículo y para la realización de estudios sobre la lectura y las bibliotecas escolares, convocadas por Orden ECI/2687/2007, de 6 de septiembre, Modalidad C.

\section{Bibliografía}

Bundy, A. (1998). Information Literacy: The Key Competency for the $21^{\text {st }}$ Century [en línea]. En: Proceedings of the IATUL Conference. Pretoria, Surafrica, 1-5 de junio de 1998. <http://arrow.unisa.edu.au:8081/1959.8/40613> [consulta: 13-02-2011].

Catts, R., y Lau, J. (2008). Towards information Literacy Indicators [en línea]. París: Unesco, p. $44<$ http://www.uis.unesco.org/template/pdf/cscl/InfoLit.pdf> [consulta: 13-02-2011].

Colás, P.; Rodríguez, M., y Jiménez, R. (2005). Evaluación de e-learning. Indicadores de calidad desde el enfoque sociocultural. Teoría de la Educación: Educación y Cultura en la Sociedad de la Información, vol. 6 (2), 1-11.

Driscoll, M. A. (2010). Information Literacy Seven Corners: Improving instruction by reviewing how librarians, faculty culture, professional literature, technology, and today's college students converge [en línea]. Library Student Journal, vol. 5. <http://www.librarystudentjournal.org/index.php/lsj/article/view/133/235> [consulta: 13-02-2011].

Emmet, A., y Ende, J. (2007). Assessing Information Literacy Skills using ACRL Standards as a Guide. Referente Services Review, vol. 35 (2), 210-229.

i2010 High Level Group (2009). Benchmarking Digital Europe 2011-2015: conceptual framework. [en línea]. Luxembourg: Publications Office of the European Union, p.199 $<$ http://ec.europa.eu/information_society/eeurope/i2010/docs/benchmarking/benchmarking_digital_europe_2011-2015.pdf> [consulta: 13-02-2011].

Instituto de Tecnologías Educativas (2010). Indicadores y datos de las Tecnologías de la Información y Comunicación en la Educación en Europa y España [en línea]. Madrid: ITE, Ministerio de Educación, p. 35. <http://recursostic.educacion.es/blogs/europa/ media/blogs/europa/informes/Indicadores_y_datos_de_las_TIC_en_la_\%20Educacion_ en_Europa_y_Espana_ITE_2009.pdf > [consulta: 13-02-2011].

Lindauer, B. G. (2006). Los tres ámbitos de evaluación de la alfabetización en información. Anales de Documentación, vol. 9, 69-81. 
Marzal, M. A. (2009). Evolución conceptual de la alfabetización en información a partir de la alfabetización múltiple en su perspectiva educativa y bibliotecaria. Investigación Bibliotecológica (Revista de CUIB), vol. 23, (47), 129-160.

Marzal, M. A., y Cuevas, A. (2007). Biblioteca escolar para la sociedad del conocimiento en España. Ciência da Informação, vol. 37 (1), 54-68.

Viñas, V. (2004). Evaluación cualitativa de programas de desarrollo regional en zonas rurales. Revista de Estudios Regionales, vol. 71, 13-36. 